TITLE:

\title{
Enhanced luminescence from electron-hole droplets in silicon nanolayers
}

AUTHOR(S):

Nihonyanagi, Satoshi; Kanemitsu, Yoshihiko

CITATION:

Nihonyanagi, Satoshi ...[et al]. Enhanced luminescence from electronhole droplets in silicon nanolayers. Applied Physics Letters 2004, 85(23): 5721.

\section{ISSUE DATE:}

2004-12

URL:

http://hdl.handle.net/2433/87367

RIGHT:

c 2004 American Institute of Physics. 


\title{
Enhanced luminescence from electron-hole droplets in silicon nanolayers
}

\author{
Satoshi Nihonyanagi \\ Graduate School of Materials Science, Nara Institute of Science and Technology, \\ Ikoma, Nara 630-0192, Japan \\ Yoshihiko Kanemitsu ${ }^{\text {a) }}$ \\ International Research Center for Elements Science, Institute for Chemical Research, Kyoto University, Uji, \\ Kyoto 611-0011, Japan and Graduate School of Materials Science, Nara Institute of Science and \\ Technology, Ikoma, Nara 630-0192, Japan
}

(Received 9 August 2004; accepted 5 October 2004)

\begin{abstract}
We have studied photoluminescence (PL) from the condensed phase in silicon-on-insulator samples with different Si layer thickness from 50 to $340 \mathrm{~nm}$. Two major PL bands are observed at low temperatures, originating from free excitons (FE) and electron-hole droplets (EHD). It is found that with an increase of the excitation intensity the EHD PL shows a linear increase in the 50-nm-thick layer while a superlinear increase in the 340-nm-thick layer. The intensity ratio of the EHD PL to the FE PL in the 50-nm-thick layer is much larger than that in the 340-nm-thick layer under the same experimental conditions. The luminescence from the EHD is enhanced in thin Si nanolayers. These results suggest that highly dense electrons and holes are formed in the Si nanolayer and the interfaces act as the nucleation center of the EHD. () 2004 American Institute of Physics. [DOI: $10.1063 / 1.1829161]$
\end{abstract}

In the past three decades, the condensed phase of electrons and holes in bulk Si have been extensively studied both theoretically and experimentally, and enormous and intimate knowledge about its optical properties has been stored. ${ }^{1-4}$ With an increase of electron-hole pair density in bulk $\mathrm{Si}$, free exciton (FE) gas begins to condense into liquid and forms droplets, called electron-hole droplet (EHD). The radius of the EHD is estimated to be several micrometers in the case of bulk Ge. ${ }^{5}$ The radius of the EHD in bulk Si maybe has the same order. Therefore, it is expected that the luminescence properties of the FE and EHD in nanostructures are completely different from those in bulk $\mathrm{Si}^{6,7}$ because the growth of droplets is restricted in a very small volume. However, to our knowledge, there are only a few experimental reports on properties of the condensed phase in silicon nanostructures. Recently, Tajima and Ibuka ${ }^{8}$ observed the photoluminescence (PL) from the EHD in a few-hundred-nanometer thick silicon-on-insulator (SOI) wafer under excitation intensities where the condensation does not occur in bulk Si. Very recently, Pauc et al. ${ }^{9}$ reported the PL of the EHD in the wide range of thickness of SOI layers where quantum confinement effects have dominant influences on electronic states. Nevertheless, the mechanism of the enhancement of the EHD luminescence is not clear. In this letter, we report a lowtemperature PL study of 50-340 nm Si nanolayers of SOI wafers and discuss their FE and EHD PL properties.

In large nanostructures where the sizes are much larger than the exciton Bohr radius in bulk $\mathrm{Si}(\sim 4.5 \mathrm{~nm})$, quantum confinement effects do not affect the FE spectrum; the sharp FE PL bands are observed at the near-infrared spectral region, similar to the case of bulk Si. However, the PL efficiency in large nanostructures is much higher than that of bulk $\mathrm{Si}$, and the intrinsic PL bands are clearly observed at elevated temperatures near room temperature. ${ }^{6,7}$ Therefore, it

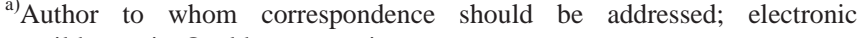
mail:kanemitu@scl.kyoto-u.ac.jp
}

is very important to investigate the FE and the EHD in large $\mathrm{Si}$ nanostructures for the design of Si-based light-emitting materials and devices. Here, we show that highly dense electrons and holes are spatially confined in the thick nanolayer, and the formation of the EHD occurs at the interface.

We prepared several Si nanolayer samples with different thickness from the identical SOI wafer. The SOI wafer had a 340-nm-thick $p$-type Si layer with a resistivity of 14-22 $\Omega \mathrm{cm}$, and a $400-\mathrm{nm}$-thick buried $\mathrm{SiO}_{2}$ layer. The SOI wafer was etched at $33{ }^{\circ} \mathrm{C}$ in a tetramethyl-ammonium hydroxide (TMAH) solution. The thickness of the Si layer was controlled by changing etching duration time, and was ranged from 50 to $340 \mathrm{~nm}$. The Si layer thickness was determined by using a cross-sectional scanning electron microscope and a spectroscopic ellipsometer.

PL measurements were performed through an objective lens, which makes possible the exact adjustment of excited positions and excitation intensities. In addition, the shallow depth of focus realized to collect efficiently the luminescence from a surface layer. An excitation light was a $488 \mathrm{~nm}$ Ar-ion cw laser and focused on the sample through an objective lens with numerical aperture of 0.42 . The typical beam size on the sample surface was carefully estimated to be about $1.5 \mu \mathrm{m}$ by a knife-edge method. The PL was also collected through the objective lens. The PL signal was dispersed by a $25 \mathrm{~cm}$ monochromator and detected by an InP/InGaAsP photomultiplier. The photomultiplier signals were integrated by a photon counting method. The samples were mounted on the cold finger of a temperature-variable closed cycle He gas cryostat. Here, PL properties of two different samples are reported in detail: the thin $50 \mathrm{~nm} \mathrm{Si}$ layer and the thick $340 \mathrm{~nm}$ Si-layer samples.

The PL spectra of the $340 \mathrm{~nm}$ Si layer at $10 \mathrm{~K}$ are shown in Fig. 1. Under low intensity excitation [solid curve (a)], three intrinsic phonon-assisted PL bands are observed: the TO phonon band $\left(I^{\mathrm{TO}}\right)$, the TA phonon band $\left(I^{\mathrm{TA}}\right)$, and the $\mathrm{TO}+\mathrm{O}^{\Gamma}$ phonon band $\left(\mathrm{I}^{\mathrm{TO}+\mathrm{O}^{\Gamma}}\right)$ appear at $1.099,1.138$, and 


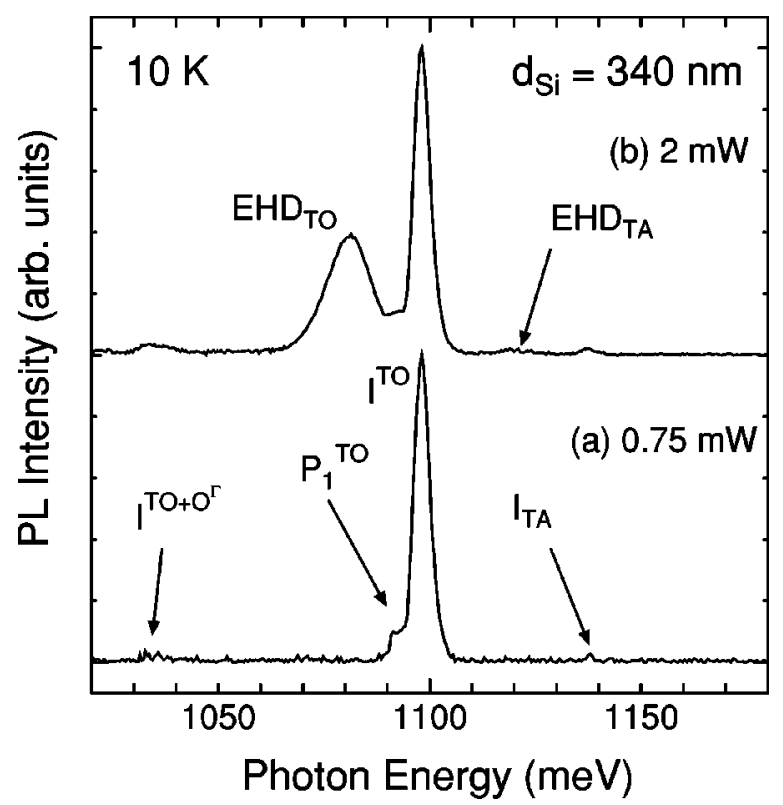

FIG. 1. PL spectra in the 340 -nm-thick Si layer under (a) $0.75 \mathrm{~mW}$ and (b) $2 \mathrm{~mW}$ excitation intensities.

$1.035 \mathrm{eV}$, respectively, where TO phonon is transverse optical phonon, TA phonon is transverse acoustic phonon, and $\mathrm{O}^{\Gamma}$ is the zero-center optical phonon selected for intervalley scattering. ${ }^{10}$ in addition, a phosphorus bound exciton $\left(P_{0}^{\mathrm{TO}}\right)$ line is observed near $1.092 \mathrm{eV}$, at the lower side of the TO phonon band. The intensity ratio of $P_{0}^{\mathrm{TO}}$ to $I^{\mathrm{TO}}$ bands implies that the impurity concentration is estimated to be lower than $1 \times 10^{12} \mathrm{~cm}^{-3} \cdot 11,12$ Therefore, we consider the Si nanolayer fabricated from the SOI layer as high purity $\mathrm{Si}$ and thus can study intrinsic luminescence properties of the Si nanolayers. Under high intensity excitation [solid curve (b)], broad bands appear around 0.8 and $1.12 \mathrm{eV}$. These two bands originate from the phonon-assisted recombination of electrons and holes in the EHD. ${ }^{2}$ The high-energy and low-energy bands are the TO-phonon-assisted EHD band $\left(\mathrm{EHD}_{\mathrm{TO}}\right)$ and the TAphonon-assisted EHD band $\left(\mathrm{EHD}_{\mathrm{TA}}\right)$, respectively. The PL from the EHD will be discussed later.

Figure 2 shows the PL spectra of the 50 and $340 \mathrm{~nm} \mathrm{Si}$ layer samples as a function of the excitation intensity at 10 $\mathrm{K}$. The excitation intensities range from 0.75 to $30 \mathrm{~mW}$, corresponding to $\sim 2 \times 10^{15}$ to $\sim 1 \times 10^{17} \mathrm{~cm}^{-3}$ in the average
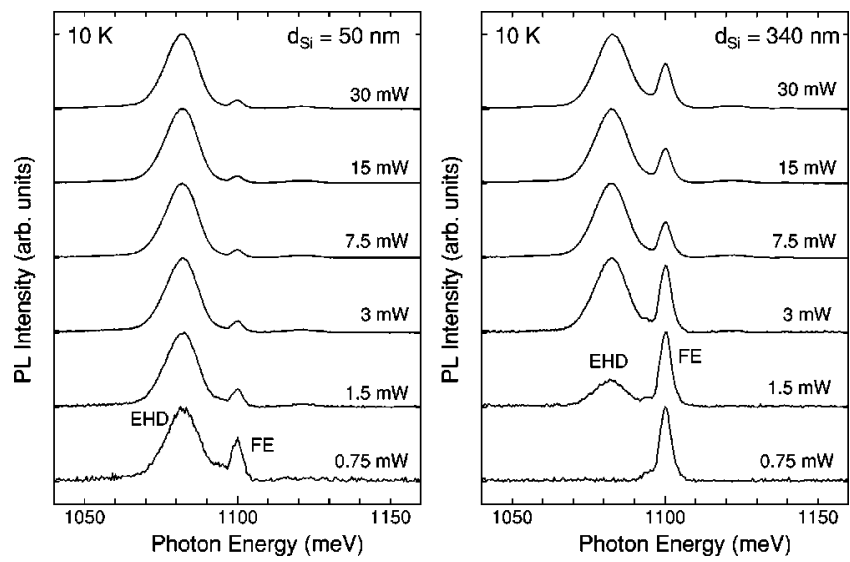

FIG. 2. PL spectra of the 50 and $340 \mathrm{~nm} \mathrm{Si} \mathrm{layer} \mathrm{samples} \mathrm{at} \mathrm{different}$

sample the efficient formation of the EHD occurs even under
Downloaded 16 Nov 2009 to 130.54.110.32. Redistribution subject to AIP license or copyright; see http://apl.aip.org/apl/copyright.jsp

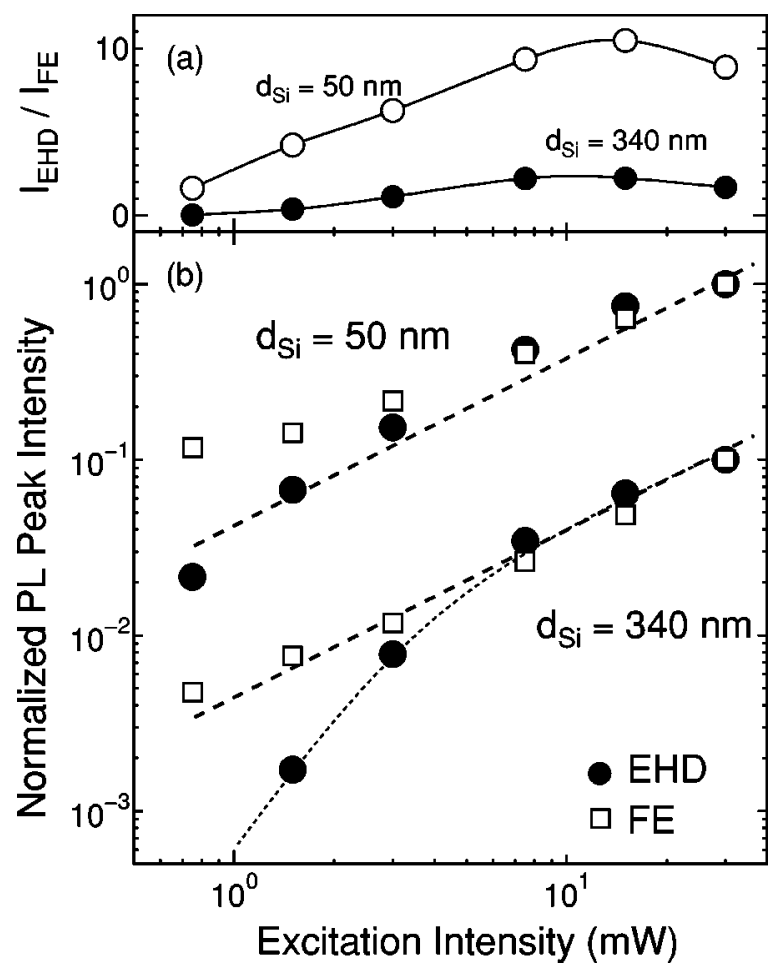

FIG. 3. (a) The excitation intensity dependence of $I_{\mathrm{EHD}} / I_{\mathrm{FE}}$. (b) The excitation intensity dependence of the peak intensity of the FE and EHD luminescence. The broken and dotted lines are guides to eyes. The broken lines show a linear dependence.

density of electron-hole pairs, respectively. ${ }^{13}$ In both samples, the luminescence of the FE and EHD are clearly observed, similar to the case of bulk Si. With an increase of the excitation intensity, the intensities of the FE and EHD luminescence increase with no shift of the peak energies because the electron-hole pair density in the EHD state is constant and estimated to be $2.9 \times 10^{18} \mathrm{~cm}^{-3}$ from theoretical fittings of PL spectra. ${ }^{2}$ Under weak intensity excitation of $0.75 \mathrm{~mW}$, the EHD luminescence is observed in the $50 \mathrm{~nm} \mathrm{Si}$ layer sample, while the EHD luminescence is not observed in the $340 \mathrm{~nm}$ Si layer sample.

Figure 3(a) shows the excitation intensity dependence of the intensity ratio of the EHD luminescence to the FE luminescence, $I_{\mathrm{EHD}} / I_{\mathrm{FE}}$. The intensity ratio $I_{\mathrm{EHD}} / I_{\mathrm{FE}}$ in the $50 \mathrm{~nm}$ Si layer is much larger than in the $340 \mathrm{~nm} \mathrm{Si} \mathrm{layer} \mathrm{in} \mathrm{all}$ excitation intensities. The reduction of the ratio under higher excitation region (e.g., $30 \mathrm{~mW}$ in the $340 \mathrm{~nm}$ Si layer) may be caused by the local heating by the laser beam.

In Fig. 3(b), the peak intensity of the FE and EHD PL bands in plotted as a function of the excitation intensity. The PL intensities are normalized by the values at $30 \mathrm{~mW}$. The broken and dotted lines are guides to the eyes. The broken lines show the linear dependence. In the $340 \mathrm{~nm}$ Si layer sample, the FE luminescence intensity increases linearly but the EHD luminescence increases superlinearly, with an increase of the excitation intensity. This behavior was also observed in bulk $\mathrm{Si}$. On the other hand, in the $50 \mathrm{~nm}$ Si layer sample, the FE luminescence intensity shows a sublinear increase under low excitation intensities, while the EHD luminescence intensity increases linearly. These observations [Figs. 3(a) and 3(b)] clearly show that in the $50 \mathrm{~nm}$ layer sample the efficient formation of the EHD occurs even under AIP license or copyright; see http://apl.aip.org/apl/copyright.jsp 

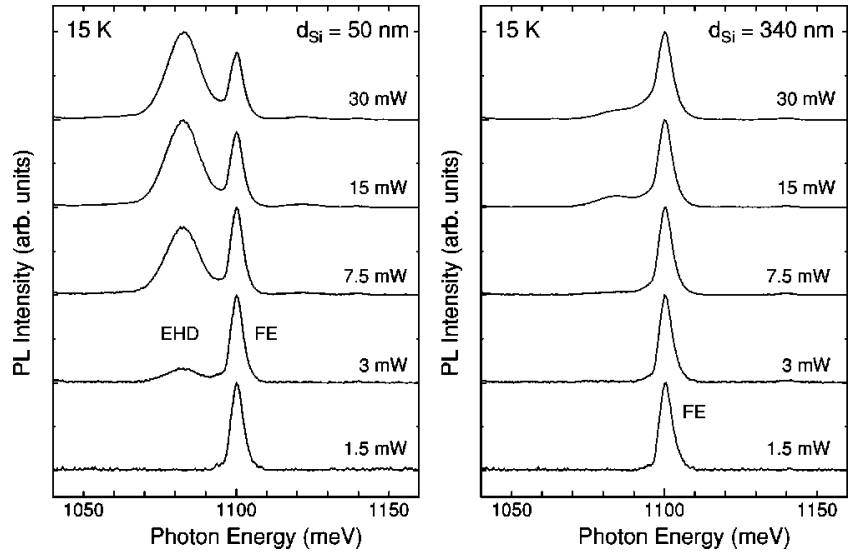

FIG. 4. PL spectra of the 50 and $340 \mathrm{~nm} \mathrm{Si}$ layer samples at different excitation intensities at $15 \mathrm{~K}$.

weak excitation, compared with the case of the thick $\mathrm{Si}$ nanolayer and bulk Si.

Figure 4 shows the excitation intensity dependence of PL spectra in both samples at an elevated temperature of 15 $\mathrm{K}$. The spectra of both samples under weak intensity excitation of $1.5 \mathrm{~mW}$ are quite similar to each other, but the spectra under strong excitation are quite different. Under $\sim 3 \mathrm{~mW}$, corresponding to $1 \times 10^{16} \mathrm{~cm}^{-3}$ in electron-hole average density, the EHD is not formed at $15 \mathrm{~K}$ in bulk Si. ${ }^{14}$ In the $340 \mathrm{~nm}$ Si layer sample, the EHD luminescence is hardly observed under $3 \mathrm{~mW}$, similar to the case of bulk Si. However, in the $50 \mathrm{~nm}$ layer sample, the EHD luminescence is clearly observed even at $15 \mathrm{~K}$ and the $I_{\mathrm{EHD}} / I_{\mathrm{FE}}$ is very large.

Let us discuss the enhancement of the EHD luminescence in thin Si nanolayers. With an increase of the average density of electron-hole pairs, $N_{e-h}$, the transition from the FE (gas) to the EHD (liquid) occurs. In the thin Si layer samples, the FE gas is spatially confined between the surface and the buried $\mathrm{SiO}_{2}$ layer. Since the diffusion coefficient, $D$, is $34 \mathrm{~cm}^{2} / \mathrm{s}$ at $20 \mathrm{~K}$ in c-Si (Refs. 15 and 16) and the PL lifetime of the FE, $\tau$, is $3.7 \mu \mathrm{s}$, the diffusion length $(D \tau)^{1 / 2}$ is estimated to be about $110 \mu \mathrm{m}$ at low temperatures. Therefore, $N_{e-h}$ in the Si layer become higher than in bulk Si since the reduction of $N_{e-h}$ by diffusion is suppressed in the nanolayer samples. This reasonably explains why the efficient formation of the EHD occurs in the Si nanolayer samples.

However, the EHD luminescence intensity in the $50 \mathrm{~nm}$ layer is much larger than that in the $340 \mathrm{~nm}$ layer. The enhancement of the EHD PL intensity in the $50 \mathrm{~nm}$ layer cannot be explained by the suppression of the carrier diffusion since the thickness of both samples is much smaller than the diffusion length. The number of photo-excited carriers per volume in the 50 and $340 \mathrm{~nm}$ layers is almost the same because the absorption depth at $488 \mathrm{~nm}$ is larger than the layer thickness. Thus, we must consider that the EHD can be formed by impurities or interfaces between $\mathrm{Si}$ and $\mathrm{SiO}_{2}$, which act as nucleation centers. ${ }^{17}$ In our two samples, the impurity concentrations are the same because the samples were fabricated from the same wafer. Then it is believed that the interface plays an important role in the enhancement of the EHD luminescence because of a large surface-to-volume ratio in thin Si layer. It is considered that semispherical drop- lets are formed on both interfaces. Taking account of the critical radius of the droplets (about $10 \mathrm{~nm}$ ),${ }^{18}$ the EHD is assumed to concentrate mainly within about $10 \mathrm{~nm}$ near the interfaces. Therefore, the ratio of the two semispherical droplets to the whole volume is up to $\sim 0.4(\simeq 20 / 50)$ in the 50nm-thick Si layer while $\sim 0.06(\simeq 20 / 350)$ in the $340 \mathrm{~nm} \mathrm{Si}$ layer. If we assume that the FE and EHD PL intensities are proportional to the volume of the FE and EHD in the layer, these estimated values show a good agreement with the observed PL intensity ratios shown in Fig. 3(a): the $I_{\mathrm{EHD}} / I_{\mathrm{FE}}$ in the $50 \mathrm{~nm}$ Si layer is about five times as large as that in the $340 \mathrm{~nm}$ Si layer. The PL spectra at $15 \mathrm{~K}$ shown in Fig. 4 also support the importance of the interfaces; in the $50 \mathrm{~nm} \mathrm{Si}$ layer the EHD luminescence is clearly observed because the formation of the droplets occurs at the interfaces.

In conclusion, we have studied the FE and EHD luminescence in Si nanolayers. It is found that in the 50-nm-thick Si layer the EHD luminescence is clearly observed even under weak intensities and even at high temperatures, where the EHD luminescence is not observed in the $340-\mathrm{nm}$-thick $\mathrm{Si}$ layer. In addition, the intensity ratio of the EHD to the FE bands in the thin Si layer is much larger than that in the thick Si layer. It is concluded that the interfaces play an important role in formation of the EHD because the thin Si layer has a large surface-to-volume ratio.

The authors would like to thank Dr. T. Tokuda of Nara Institute of Science and Technology for fruitful advice on the sample preparation. This work was supported in part by a Grant-in-Aid for Scientific Research from Japan Society for the Promotion of Science and The Research Foundation for Opto-Science and Technology.

${ }^{1}$ C. D. Jaffries, Science 189, 955 (1975).

${ }^{2}$ R. B. Hammond, T. C. McGill, and J. W. Mayer, Phys. Rev. B 13, 3566 (1976).

${ }^{3}$ Excitons, edited by E. I. Rashiva and M. D. Sturge (North-Holland, Amsterdam, 1982)

${ }^{4}$ C. Jeffries and L. Keldysh, Electron-Hole Droplets in Semiconductors (North-Holland, Amsterdam, 1983).

${ }^{5}$ Y. E. Pokarovskii, Phys. Status Solidi A 11, 385 (1972).

${ }^{6}$ Y. Kanemitsu, S. Nihonyanagi, H. Sato, and Y. Hirai, Phys. Status Solidi A 190, 755 (2002).

${ }^{7}$ S. Nihonyanagi and Y. Kanemitsu, Physica E (Amsterdam) 17, 183 (2003).

${ }^{8}$ M. Tajima and S. Ibuka, J. Appl. Phys. 84, 2224 (1998).

${ }^{9}$ N. Pauc, V. Calvo, J. Eymery, F. Fournel, and N. Magnea, Phys. Rev. Lett. 92, 236802 (2004).

${ }^{10}$ P. J. Dean, J. R. Haynes, and W. F. Flood, Phys. Rev. 161, 711 (1967).

${ }^{11}$ I. Broussell, J. A. H. Stotz, and M. L. W. Thewalt, J. Appl. Phys. 92, 5913 (2002).

${ }^{12}$ The obtained impurity concentration is two orders of magnitude lower than that estimated from the resistivity. The measurement temperature in our experiments is different from that in Ref. ${ }^{11}$.

${ }^{13}$ The average electron-hole densities were evaluated from the excitation intensity dependence of the PL intensity from the FE and EHD in bulk Si under the same experimental conditions and the phase diagram of electrons and holes in the literature [J. Shah, M. Combescot, and A. H. Dayen, Phys. Rev. Lett. 38, 1497 (1977)].

${ }^{14}$ J. Shah, M. Combescot, and A. H. Dayem, Phys. Rev. Lett. 38, 1497 (1977).

${ }^{15}$ G. Davis, Phys. Rep. 176, 84 (1989).

${ }^{16}$ J. D. Cuthbert, Phys. Rev. B 1, 1552 (1970).

${ }^{17}$ R. N. Sliver, Phys. Rev. B 11, 1569 (1975).

${ }^{18}$ H. Nakashima and Y. Shiraki, Solid State Commun. 40, 195 (1981). 